\title{
OPEN Using electrical impedance tomography for rapid determination of starch and soluble sugar contents in Rosa hybrida
}

\author{
Ji Qian ${ }^{1 \bowtie}$, Juan Zhou ${ }^{2,4}$, Bao Di ${ }^{1}$, Yang Liu ${ }^{3,4}$, Gang Zhang ${ }^{1}$ \& Xin Yang ${ }^{2,4}$
}

Soluble sugars and starches are important metabolites of plant life and physiological markers of plant stress response. There is an urgent need to develop a non-destructive and rapid method for determining plant starch and soluble sugar contents. Electrical impedance tomography (EIT) technology has been used to determine the physiological state and cold resistance of select plant tissues. However, so far there have been no reports on the use of EIT for the rapid estimation of soluble sugar and starch contents. In this study, EIT was used to obtain reconstructed voltage values and estimate starch and soluble sugar contents in the stems of three Rosa hybrida cultivars during February to May, which were grown in the Specimen Park ( $38^{\circ} 50^{\prime} \mathrm{N}, 115^{\circ} 26^{\prime} \mathrm{E}$ ) of Hebei Agricultural University, Baoding City, Hebei Province, China. Stems from two of the cultivars were used for establishing regression models for starch and soluble sugar contents as functions of reconstructed voltage values. The third cultivar was used to test the accuracy of the regression models. The quadratic regression model was best for determining soluble sugar content and the logarithmic regression model was best for determining starch content. Thus, this research provided technical support for using EIT to analyze changes in physiological parameters and to rapidly estimate physiological indexes of plants. More studies were now needed to validate the results in this paper.

Soluble sugar and starch, the photosynthetic products of higher plants, are the main substances produced through plant carbon metabolism via the Calvin cycle and play an important role in osmotic adjustment. To a certain extent, changes in soluble sugar content reflect the adaptability of a plant to environment change ${ }^{1-4}$. Wang et al. (2016) showed that during the dehardening period, as the temperature naturally changed, the soluble sugar content in rose plants decreased and the starch content increased ${ }^{5}$. At present, starch content and soluble sugar content are usually determined using colorimetric approaches. Colorimetric approaches are easily affected by the amount of chromogenic agents, proportion of different carbohydrates in the plant, and the experimental procedures and conditions. Although measurement conditions have been optimized ${ }^{6-8}$, there are still shortcomings of such methods. These shortcomings include destructive sampling, extensive use of chemicals, complicated procedures, and errors caused by human operation. Therefore, it is urgent to develop a non-destructive and rapid method for determining starch and soluble sugar contents in plants.

In 1976, Swanson from the Wisconsin Madison University in the United States, first proposed the use of electrical impedance tomography (EIT), which has since attracted extensive attention. The EIT system can quickly and efficiently detect changes in the electrical characteristics of an organism, which are associated with changes in physiological state. The voltage and current across sections of the subject are measured at the surface and the internal conductivity distribution is reconstructed. To do this, a set of EIT sensors are attached around the surface of the object of study and an electric signal with an alternating current of constant amplitude is applied to the object. An electrical instrument connected to the sensors is used to measure the boundary voltage, which is then processed and output as an intuitive two-dimensional color image $e^{9-12}$.

EIT is mainly used in animal experiments and tentatively in human clinical research related to digestive, respiratory, and craniocerebral issues ${ }^{13-17}$. In recent years, some researchers have used EIT technology to study soil properties, wood decay, root physiological state, and cold resistance of plants ${ }^{18-22}$. There are few reports

${ }^{1}$ College of Horticulture, Hebei Agricultural University, Lekai South Street 2596, Baoding 071000, Hebei, China. ${ }^{2}$ College of Electrical and Mechanical Engineering, Hebei Agricultural University, Baoding 071000, Hebei, China. ${ }^{3}$ Department of Software Engineering, Hebei Software Institute, Baoding 071000, Hebei, China. ${ }^{4}$ These authors contributed equally: Juan Zhou, Yang Liu and Xin Yang. ${ }^{\circledR}$ email: Qianji167@163.com 

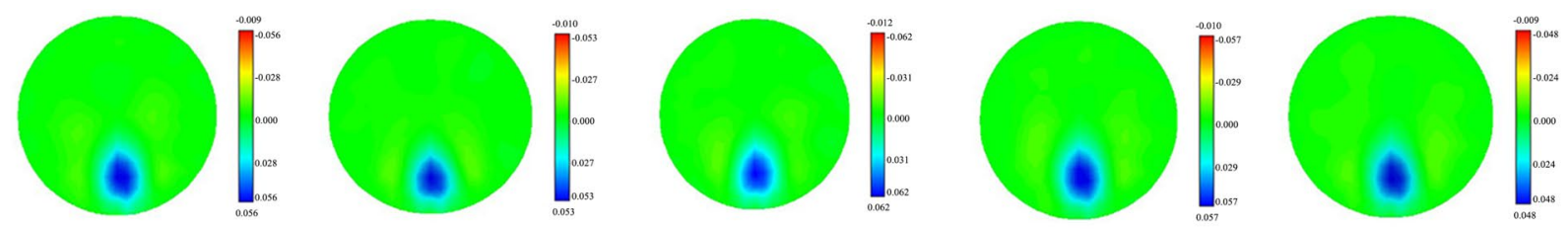

\section{'Red Cap'}

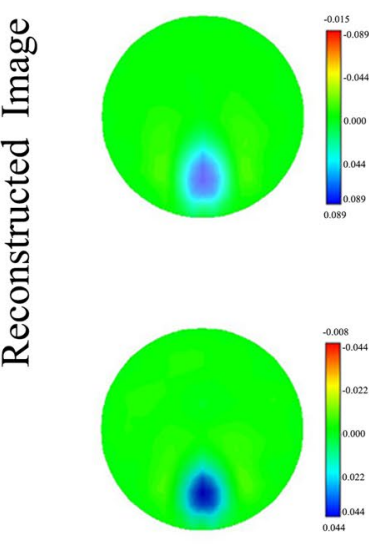

Feb 22
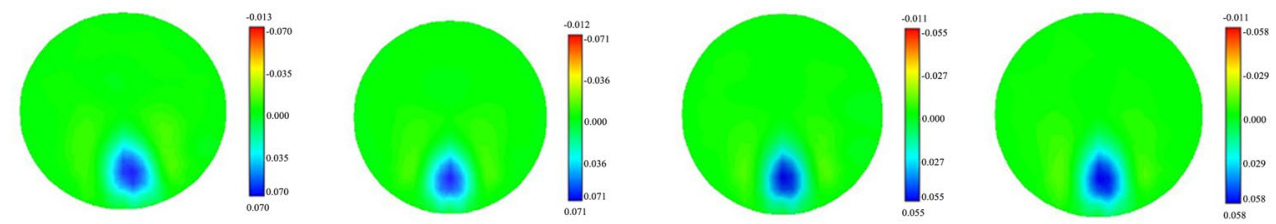

\section{'Tender and Soft as Water'}
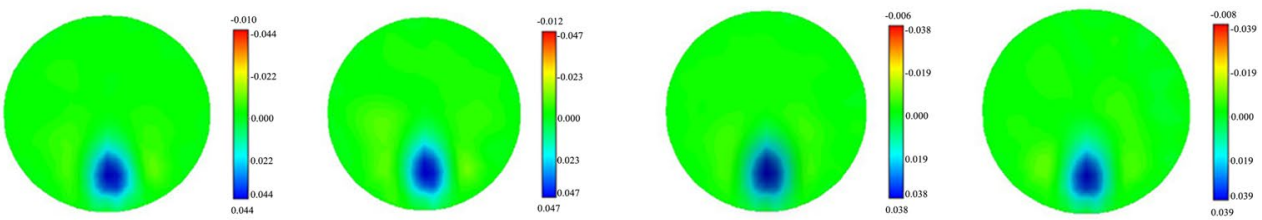

\section{'Carefree Wonder'}

Mar 14

Apr 4

Apr 25

May 16

\section{Date}

Figure 1. Electrical impedance tomography (EIT) images of the stems of three floribunda rose (Rosa hybrida) cultivars during the dehardening period.

on the establishment of regression models for the effect of plant physiological state on EIT parameters. EIT technology can show that the impedance changes with frequency inside the object and be used to display an image of the functional state of an organ undergoing physiological changes ${ }^{23}$. The rapid and non-destructive determination of plant starch and soluble sugar contents can be achieved through the following procedure: the excitation signal is loaded to the object by selecting suitable electrodes; the surface voltage is detected; data are collected and processed; the image is reconstructed using algorithms; and the corresponding two-dimensional color image is displayed on a computer.

We hypothesized that the starch and soluble sugar contents in plant cells and tissues would change during the dehardening period ${ }^{24}$. The resulting differences in electrical conductivity could be detected using EIT technology by measuring the boundary voltage. Hence, the concentrations of the soluble sugar and starch contents could be detected. The objective of this study was to utilize the EIT imaging technology to establish regression models for the starch and soluble sugar contents as functions of reconstructed EIT boundary voltage values using data from two floribunda rose (Rosa hybrida) cultivars. The accuracy of the models was tested using a third floribunda rose cultivar. This study provides a technical and theoretical basis, and a mathematical model, for the rapid and non-destructive determination of starch and soluble sugar contents in plants.

\section{Results}

EIT images and their reconstructed boundary voltage values. One EIT image was selected from each of the three rose cultivars at different temperatures at each sampling time (Fig. 1). During the period of external temperature change, the EIT image accurately displayed the location, shape, and size of the rose stem as well as the distribution of resistivity. The darker the blue color, the smaller the resistivity and the corresponding reconstructed boundary voltage value. In EIT images, the pure blue color corresponds to the maximum value of the reconstructed result. Over the course of the dehardening period, the blue parts of the EIT images of the stems of the three rose cultivars gradually darkened. Figure 2 showed the change of the reconstructed EIT boundary voltage value of the stems of the three rose cultivars during the dehardening period. As shown in Fig. 2, the reconstructed boundary voltage values of the stems of the three rose cultivars showed a significant downward trend. The slopes of the curves of 'Red Cap', 'Tender and Soft as Water', and 'Carefree Wonder' were $-8.84,-8.89$, and -9.20 respectively. Of these, the downward trend of 'Carefree Wonder' was the most significant and the reconstructed boundary voltage value of 'Carefree Wonder' was between 0.078 and 0.036 (Fig. 2). There were no significant differences in the reconstructed boundary voltage values between the three cultivars $(P<0.05)$.

Changes in soluble sugar and starch contents. The soluble sugar content in the stems measured with a colorimetric anthrone method. During the dehardening period, the soluble sugar content in the stems of the 


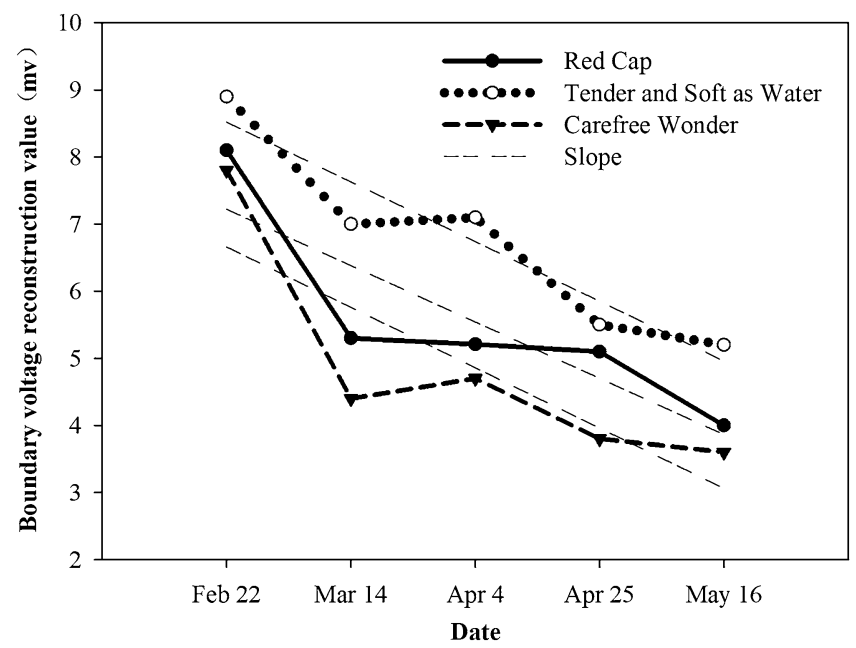

Figure 2. Reconstructed values of electrical impedance tomography (EIT) boundary voltages of the stems of three floribunda rose (Rosa hybrida) cultivars during the dehardening period.

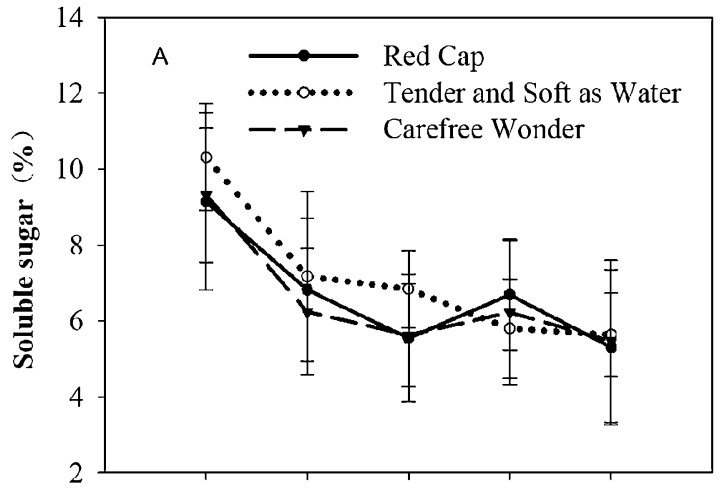

Feb 22 Mar 14 Apr 4 Apr 25 May 16

Date

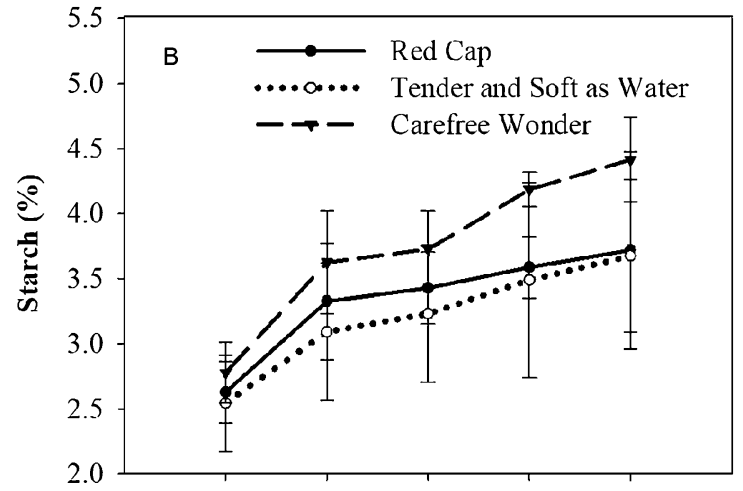

Feb 22 Mar 14 Apr 4 Apr 25 May 16 Date

Figure 3. Soluble sugar content (A) and starch content (B) in the stems of three floribunda rose (Rosa hybrida) cultivars under natural conditions during the dehardening period.

three rose cultivars showed an overall downward trend. The soluble sugar contents all increased slightly on April 25 , and then continuously decreased, with the lowest values observed on May 16 when the last sampling was performed (Fig. 3A). The soluble sugar contents of each of the three rose cultivars were significantly lower on May 16 than at the other four sampling times $(P<0.01)$. The soluble sugar contents of 'Red Cap', 'Tender and Soft as Water', and 'Carefree Wonder' on May 16 were 41.99\%, 45.38\%, and 41.35\% lower, respectively, than on February 22. However, there were no significant differences in the soluble sugar contents of the same cultivar between the March 14, April 4, and April 25 samples. Furthermore, there were no significant differences in the soluble sugar contents of the different cultivars at each sampling time.

During the dehardening period, the starch content of the stems of all three rose cultivars showed an increasing trend (Fig. 3B). The overall rate of increase in starch content of 'Carefree Wonder' was higher than that of the other two cultivars. The starch content of all three cultivars obviously increased during the period from February 22 to March 14, but the rate of increase was lower for the rest of the sampling period. The starch content of all three cultivars was significantly higher on May 16 than on the four other sampling dates $(P<0.05)$. The starch contents in the stems of 'Red Cap,' 'Tender and Soft as Water', and 'Carefree Wonder' were $41.42 \%, 44.51 \%$, and $58.93 \%$ higher on May 16 than on February 22. The starch content of the stem of 'Carefree Wonder' significantly differed between the five sampling times $(P<0.05)$. The starch contents of 'Red Cap' and 'Tender and Soft as Water' significantly differed between February 22 and the four other sampling times $(P<0.05)$; there were no significant differences in sugar content between those four sampling times. There was no significant difference in the starch content between different cultivars at each sampling time.

Establishment of the regression models for starch and soluble sugar contents as functions of reconstructed EIT boundary voltage values. The soluble sugar and starch contents and the corresponding reconstructed EIT boundary voltage values of the two cultivars 'Red Cap' and 'Carefree Wonder' were 


\begin{tabular}{|l|l|l|l|l|l|}
\hline Item & Index & Equation & Regression model & $\boldsymbol{R}^{2}$ & $\boldsymbol{F}$ \\
\hline \multirow{3}{*}{ Soluble sugar value } & \multirow{3}{*}{ Boundary voltage reconstruction value } & Linear & $y=0.868 x+2.125$ & 0.863 & 50.527 \\
\cline { 3 - 6 } & & Logarithmic & $y=4.758 \ln (x)-1.034$ & 0.811 & 34.411 \\
\cline { 3 - 6 } & & Quadratic & $y=0.142 x^{2}-0.823 x+6.757$ & 0.897 & 30.538 \\
\hline \multirow{3}{*}{ Starch value } & \multirow{3}{*}{ Boundary voltage reconstruction value } & Linear & $y=-0.333 x+5.271$ & 0.894 & 67.350 \\
\cline { 3 - 6 } & & Logarithmic & $y=-1.917 \ln (x)+6.634$ & 0.959 & 104.964 \\
\cline { 3 - 6 } & & Quadratic & $y=0.059 x^{2}-1.032 x+7.189$ & 0.935 & 50.132 \\
\hline
\end{tabular}

Table 1. Analysis of the regression for soluble sugar and starch contents as functions of reconstructed electrical impedance tomography (EIT) boundary voltage values.

\begin{tabular}{|l|l|l|l|l|l|l|}
\hline Item & Index & Regression model & $R M S E$ & $R E(\%)$ & Offset & $R^{\mathbf{2}}$ \\
\hline \multirow{4}{*}{ Soluble sugar } & \multirow{3}{*}{ Boundary voltage } & $y=0.868 x+2.125$ & 1.060 & 11.56 & 3.294 & 0.923 \\
\cline { 3 - 7 } & & $y=4.758 \ln (x)-1.034$ & 1.197 & 11.26 & 4.293 & 0.876 \\
\cline { 3 - 7 } & & $y=0.142 x^{2}-0.823 x+6.757$ & 0.811 & 10.64 & 1.380 & 0.977 \\
\hline \multirow{3}{*}{ Starch } & \multirow{3}{*}{ Boundary voltage } & $y=-0.333 x+5.271$ & 0.202 & -5.58 & 0.554 & 0.926 \\
\cline { 3 - 7 } & & $y=-1.917 \ln (x)+6.634$ & 0.212 & -6.02 & 0.022 & 0.948 \\
\cline { 3 - 8 } & & $y=0.059 x^{2}-1.032 x+7.189$ & 0.257 & -5.88 & 0.861 & 0.824 \\
\hline
\end{tabular}

Table 2. Indexes for assessing the fitting of measured and predicted values of soluble sugar and starch contents.

used to establish a regression model. The measured EIT boundary voltage values ranged from 0.036 to 0.089 . These values were 100 times smaller than the values of starch and soluble sugar contents. Therefore, the measured boundary voltage values were multiplied by 100 . These amplified values were used together with the soluble sugar and starch contents in linear and nonlinear regression analyses. The regression model with the greatest $R^{2}$ was preferentially selected as the best model. The results are shown in Table 1 . The $R^{2}$ values of the established linear, logarithmic, and quadratic regression models for soluble sugar content as a function of reconstructed EIT boundary voltage value, were $0.863,0.811$, and 0.897 , respectively $(P<0.001)$. Thus, for soluble sugar, the quadratic regression model had the greatest $R^{2}$. The $R^{2}$ values of the established linear, logarithmic, and quadratic regression models for the starch content as a function of reconstructed EIT boundary voltage value, were 0.894, 0.959 , and 0.935 , respectively $(P<0.001)$. Thus, for starch, the logarithmic regression model had the greatest $R^{2}$.

Testing the accuracy of the regression models for soluble sugar and starch contents as functions of reconstructed EIT boundary voltage values. The measured data from the cultivar 'Tender and Soft as Water' was used to test the accuracy of the regression models. The Root Mean Square Error (RMSE), Relative Error $(R E)$, Offset, and the coefficient of determination $\left(R^{\prime 2}\right)$ of the fitted linear regression based on measured and predicted values were compared between the established linear, logarithmic, and quadratic models. The $R E$ is the percentage of the absolute error of the measurement divided by the measured value. Generally, the $R E$ could well reflect the reliability of a measurement and was calculated using the Eq. (1). RMSE was used to estimate the deviation between the predicted value and the measured value ${ }^{25}$, and was usually used as a standard for assessing the predicted results of a model. The RMSE was calculated using Eq. (2).

$$
\begin{gathered}
R E=\frac{\sum_{i=1}^{n} f\left(x_{i}\right)-\sum_{i=1}^{n} y_{i}}{\sum_{i=1}^{n} y_{i}} \times 100 \% \\
R M S E=\sqrt{\frac{1}{n} \sum_{i=1}^{n}\left(f\left(x_{i}\right)-y_{i}\right)^{2}}
\end{gathered}
$$

where $x_{\mathrm{i}}$ refers to the measured reconstructed EIT voltage values of 'Tender and Soft as Water'; $f\left(x_{\mathrm{i}}\right)$ was the soluble sugar content and starch content calculated by the model; $y_{\mathrm{i}}$ was the measured value of soluble sugar and starch content corresponding to $x_{\mathrm{i}}$; and $n=5$ represents the five sampling times within the dehardening period.

The validation result of the models was shown in Table 2 . The quadratic regression model for soluble sugar content prediction $\left(y=0.142 x^{2}-0.823 x+6.757\right)$ had the greatest $R^{2}(0.897)$, and the smallest $R M S E(0.811)$, $R E(10.64 \%)$, and Offset (1.38). Therefore, it was the best model and the prediction accuracy was $89.36 \%$. The logarithmic regression model for starch content prediction $(y=-1.917 \ln (x)+6.634)$ had the greatest $R^{2}(0.959)$ and $R^{\prime 2}(0.948)$, and the smallest Offset (0.022). The RMSE was only 0.010 times greater than that of the linear 
model. Taking into consideration the $R^{2}$, RMSE, RE, Offset, and $R^{2}$, the logarithmic model was the best model for predicting starch content, with a prediction accuracy of $93.98 \%$.

\section{Discussion}

Carbohydrates, which exist mainly as starch and soluble sugar, were the basic substances that directly participated in plant metabolism by providing energy and carbon sources. Soluble sugars included glucose, fructose, sucrose, etc. The accumulation of starch in plants was closely related to the synthesis, transport and accumulation of soluble sugar. Thus, they were the important indicators of the intensity of life activities in plants. Many studies had shown that carbohydrates could also act as signaling molecules, connecting with hormone and nitrogen signals to form a complex signaling network. Thus, carbohydrates participated in the regulation of a series of plant metabolic activities. Carbohydrate content in plants directly affected plant growth and ecological adaptability ${ }^{26-29}$. In the present study, it could be seen that during the dehardening period, large amounts of soluble sugar were used for growth and the content of soluble sugar in the rose stems showed a decreasing trend. As the environmental temperature and duration of sunshine increases, more photosynthetic products were produced by new green leaves ${ }^{22,30}$. Meanwhile, the activities of various enzymes involved in photosynthesis were enhanced. More photosynthetic products (soluble sugars) are produced, and most of them are synthesized into starch, leading to an increase in starch content. As a result, the starch content in the stem increases, as was observed in the present study. Therefore, the content of soluble sugar and starch in the plant changed with the temperature of the external environment.

EIT technology used the electromagnetic characteristics of a tissue to reconstruct the distribution of resistivity inside the tissue and display these changed as an image. The interaction of charged ions and ionic groups allowed biological tissues to maintain a certain structure and function. Changes in physiological state result in changes to the electrical characteristics, which could be sensitively captured by the EIT system. Therefore, EIT was an imaging technology that could reflect the internal structure and function of tissues and organs. In response to changes in the external environment, plants presented a series of noticeable changes, such as in plasma membrane permeability, intracellular solutes, and cell growth and differentiation ${ }^{31}$. In the present study, the stem segment was exposed to the EIT saline solution for only $30 \mathrm{~s}$, so the effect of the water content on the rose stem boundary voltage could be ignored.

Rose stems mainly possess fiber wood-related characteristics, which were affected by the changes in physiological parameters and in turn led to the changes in the stem dielectric properties. These dielectric changes caused differences in the reconstructed boundary voltage values. Rose stems were composed of a large number of cells of different shapes. The cells had high impedance to low frequency currents $(\mathrm{f}<1 \mathrm{kHz})$ that only flow in the extracellular space ${ }^{32}$. In the present study, we used $1 \mathrm{kHz}$ excitation currents. The majority of the current flows only in the extracellular spaces, and only a small part of the current passed through the cell membrane into the cell. Therefore, the extracellular impedance and part of the intracellular impedance affected the reconstructed EIT boundary voltage value.

Sugar dissolves in the cell fluid, while starch was insoluble in water. As the environmental temperature gradually increases, the carbohydrates in the stem changed accordingly. In the present study, the soluble sugar content gradually decreased while the starch content gradually increased. This affected the flow of the electric current. As a result, the stem resistivity and the reconstructed EIT boundary voltage value decreased over the course of the dehardening period. It was found that the soluble sugar and starch contents correlated well with the reconstructed EIT boundary voltage value. The $R^{2}$ of the best regression models for predicting soluble sugar content and for predicting starch content were 0.897 and 0.959 , respectively $(P<0.001)$. In addition, by using rose stem samples from a third cultivar for testing, both of the models were found to have decent accuracy. Therefore, it would be possible to use the reconstructed EIT boundary voltage value to predict plant soluble sugar and starch contents as a substitute for cumbersome chemical methods.

More studies were now needed to validate the results in this paper.

\section{Conclusions}

The rapid advancement of EIT technology deems it feasible for use as a rapid and non-destructive method for the determination of physiological parameters of plants. In this study, the mathematical models for estimating soluble sugar and starch contents were preliminarily explored using the reconstructed EIT boundary voltage value of rose stems. The results provide theoretical and technical basis for the application of EIT technology in the rapid analysis of the physiological parameters of plants and their changes. This study may also promote the application of EIT technology for physiological studies of plant resistance. More studies wrer now needed to validate the results in this paper.

\section{Methods}

Rose plants, growth conditions, and sampling. The biennial floribunda rose ( $R$. hybrida) cultivars 'Red Cap', 'Tender and Soft as Water', and 'Carefree Wonder', which were grown in the Specimen Park $\left(38^{\circ} 50^{\prime}\right.$ $\mathrm{N}, 115^{\circ} 26^{\prime} \mathrm{E}$ ) of Hebei Agricultural University, Baoding City, Hebei Province, China, were used in the study. A completely randomized block design was adopted. Before the roses were planted, the soil was ploughed and barnyard manure fertilizer was applied. After planting, the soil was watered one to two times per week according to rainfall conditions. Thirty plants were grown in each plot with a spacing of $0.25 \mathrm{~m} \times 0.30 \mathrm{~m}$ between plants. There were five replicate plots for each cultivar. A total of 450 plants were grown ( 30 plants/plot $\times 5$ replicates $\times 3$ cultivars $=450$ plants). Management was applied to maintain consistent growth of the different rose cultivars. During the dehardening period from February to May 2016, the plants were sampled every 20 days and five times in total. During the sampling period, the environmental temperature was recorded by the meteorologi- 


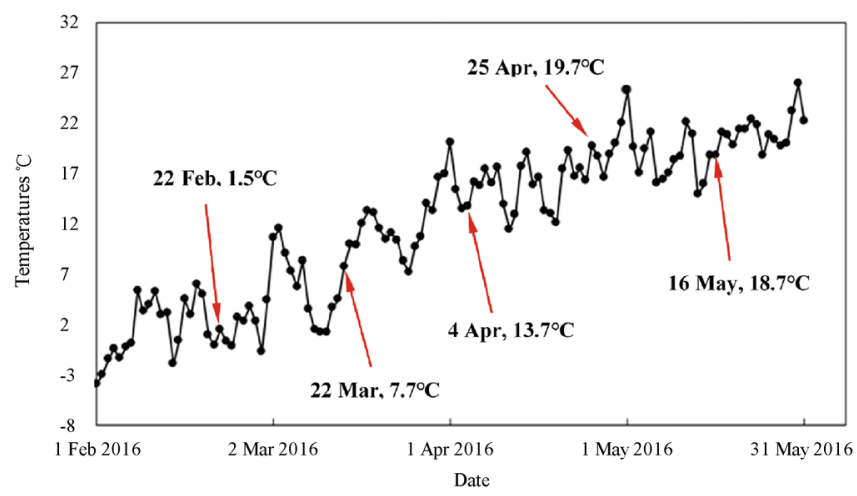

Figure 4. Changes in environmental temperature during the sampling period.

cal station in the Specimen Park of Hebei Agricultural University. The daily average air temperatures of the five sampling days were $1.45^{\circ} \mathrm{C}, 7.7^{\circ} \mathrm{C}, 13.65^{\circ} \mathrm{C}, 19.65^{\circ} \mathrm{C}$, and $18.75^{\circ} \mathrm{C}$ (Fig. 4 ).

Five plants which grew well were selected from each cultivar. Three to four branches $(1 \mathrm{~cm}$ diameter $)$ with leaves, from the middle of each plant, were sampled. The samples were washed three times with tap water and a further three times with deionized water. The water on the surface of the samples was removed with absorbent papers. A part of each sample was stored in a refrigerator at $4{ }^{\circ} \mathrm{C}$ for $2 \mathrm{~h}$ and then used in the EIT measurement. The other part of each sample was oven dried for the conventional determination of starch and soluble sugar contents.

Determination of reconstructed EIT boundary voltage value. In this study, we used the relatively high precision EIT system that was independently developed by researchers from the Fourth Military Medical University, Xi'an City, Shaanxi Province, China. A number of preliminary tests were conducted in order to find the excitation current that provided the best imaging and avoided noise interference. The chosen excitation current for the EIT system in this study was $1 \mathrm{kHz}$ and $250 \mu \mathrm{A}$. A cylindrical perspex container with an inner diameter of $80 \mathrm{~mm}$ and a height of $80 \mathrm{~mm}$ was filled with $0.9 \%$ physiological saline solution (impedance does not change with frequency). Sixteen electrodes of $10 \mathrm{~mm}$ diameter were equidistantly installed on the wall inside the container. The model of relative excitation and adjacent measurement was adopted. The electrode plane was $25 \mathrm{~mm}$ from the bottom of the container and $10 \mathrm{~mm}$ below the surface of the physiological saline solution. Stem segments that were $70-90 \mathrm{~mm}$ long and $8-10 \mathrm{~mm}$ in diameter (excessively thin segments downgraded the image quality) with even thickness and growth status were selected. Separately, each of the five stem segments was inserted vertically in the container, $20 \mathrm{~mm}$ away from the wall. After the surface of the saline solution became still, 10 frames of EIT images were obtained from each stem segment. Of these, the image with the least noise interference was selected and the corresponding reconstructed boundary voltage value was used as the final datum. For the EIT, a reconstruction algorithm was used to divide the image region into several triangular elements. A total of 512 finite elements were used for image reconstruction. To improve the visual display effect, the image was smoothed. EIT imaging results are displayed using a relative gray scale: in the obtained images, a reconstruction value less than 0 indicated that there was a reduction in resistivity of the corresponding area. In the pseudo-color images, negative reconstruction values were represented by colors on the red end of the scale, and the pure red part of the image corresponded to the minimum EIT reconstruction value obtained. A reconstruction value greater than 0 indicated that there was an increase in resistivity of the corresponding region, which was represented by colors towards the blue end of the scale. The pure blue part of the image corresponded to the maximum reconstruction value. The median reconstruction value corresponded to the pure green color shown in the image. According to the algorithm, the greater the absolute value of the reconstruction results at a location, the greater the degree of variation in resistivity of the region.

The entire data acquisition process took only $30 \mathrm{~s}$. In such a short time, the effect of the saline solution on the stem segment and the subsequent effect on the precision of the image could be ignored. In order to obtain the best quality image, we used the weighted and damped least-squares (WD-DLS) based EIT algorithm to reconstruct the image. This method was used for data processing, which eliminated the influence of background noise on the imaging quality. Meanwhile, the regularization method combined with the damped least-square reconstruction algorithm was used to deal with the ill-posed inverse problem associated with EIT. The structure of the system is illustrated in Fig. 5.

Determination of soluble sugar and starch contents. The sampled stem segments were washed with deionized water, oven dried at $80^{\circ} \mathrm{C}$ for $48 \mathrm{~h}$, and then kept in desiccators before use. Soluble sugar and starch contents were determined using the anthrone colorimetric method ${ }^{33}$. Eight samples from each replicate were used.

To the precipitate in the tube, $2 \mathrm{~mL}$ water was added and the two were mixed by stirring. The tube was placed in boiling water for $15 \mathrm{~min}$, and the precipitate formed a paste. After cooling on ice, $2 \mathrm{~mL}$ of $9.2 \mathrm{~mol} / \mathrm{L}$ perchloric acid was added and the mixture was stirred well. After $15 \mathrm{~min}, 3 \mathrm{~mL}$ water was added, and the mixture was stirred 


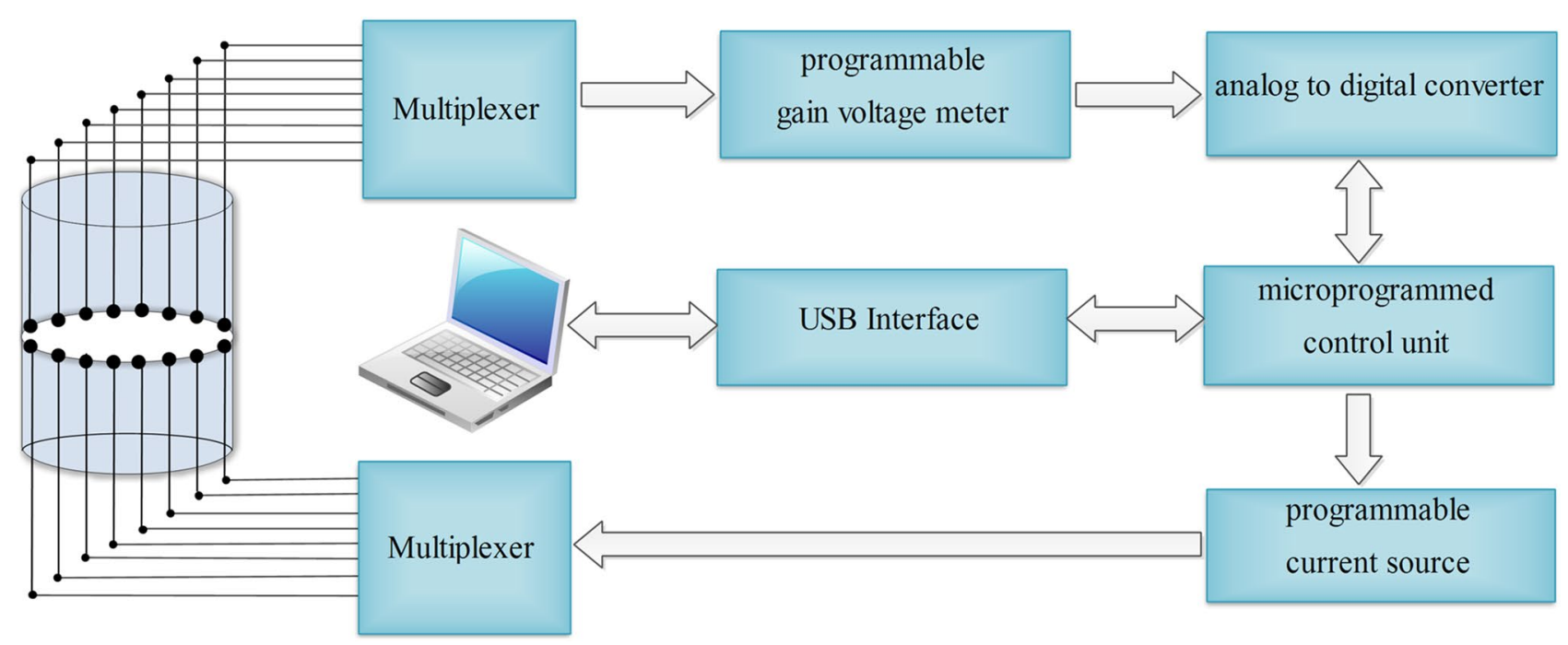

Figure 5. Diagram of the electrical impedance tomography (EIT) data acquisition syst.

well. The tube was then centrifuged for $10 \mathrm{~min}$. The supernatant was poured into a $50 \mathrm{~mL}$ volumetric flask. To the precipitate in the tube, $2 \mathrm{~mL}$ of $4.6 \mathrm{~mol} / \mathrm{L}$ perchloric acid was added and the mixture was stirred well. After $15 \mathrm{~min}, 3 \mathrm{~mL}$ of water was added, the mixture was stirred well, and then centrifuged for $10 \mathrm{~min}$. This supernatant was also poured into the $50 \mathrm{~mL}$ volumetric flask. Finally, the precipitate was washed with $4 \mathrm{~mL}$ water and centrifuged. The centrifuged supernatant was combined with the previous supernatants collected in the $50 \mathrm{~mL}$ volumetric flask. Distilled water was added to make a final volume of $50 \mathrm{~mL}$.

In a clean test tube, $1 \mathrm{~mL}$ water, and then $1 \mathrm{~mL}$ of extract (centrifuged supernatants collected in the $50 \mathrm{~mL}$ flask) were added. For the control, the test tube contained $2 \mathrm{~mL}$ water. Along the wall of the test tube, $5 \mathrm{~mL}$ $0.2 \%$ anthrone was slowly added, and the solution in the tube was mixed by shaking. The test tube was then incubated in an $80^{\circ} \mathrm{C}$ water bath for $10 \mathrm{~min}$ to allow for color formation. After being cooled, the absorbance of the solution was measured at $620 \mathrm{~nm}$. The soluble sugar and starch contents were calculated according to the following Eqs. (3) and (4):

$$
\begin{aligned}
& \text { Soluble sugar }(\%)=\frac{C \cdot n \cdot(V / \alpha)}{W \times 1000} \times 100 \% \\
& \text { Starch }(\%)=\frac{C \cdot n \cdot(V / \alpha) \times 0.9}{W \times 1000} \times 100 \%
\end{aligned}
$$

where $C$ is the glucose content $(\mu \mathrm{g})$ of the tested sample in the cuvette, which was read from a standard curve; $V$ represents the total volume of extracts $(\mathrm{mL}) ; \alpha$ represents the volume of extracts $(\mathrm{mL})$ used in displaying color; $n$ represents the fold of dilution; $W$ is the dry weight of the sample $(\mathrm{mg})$; and 0.9 is the glucose to starch conversion constant.

Statistical analysis. Microsoft Excel 2016 was used to remove anomalous data according to error theory ${ }^{34}$. The curves of the reconstructed EIT boundary voltage values and the soluble sugar and starch contents changing with the sampling times were plotted using SigmaPlot V 10.0 (mean values were used in the figure). The correlation and variance of reconstructed EIT boundary voltage value, soluble sugar content, and starch content between the three floribunda cultivars and between the different sampling dates of each cultivar were analyzed using SPSS V 20.0 software. SPSS (Version 22.0.0.1 https://www.ibm.com/cn-zh/analytics/spss-statistics-softw are?lnk=hmhmmpr_bua_cnzh) was used to establish the regression models for the average soluble sugar or starch content (dependent variable $y$ ) of the 'Red Cap' and 'Carefree Wonder' stems as a function of their corresponding reconstructed EIT boundary voltage values (independent variable $x$ ). The measured data for the cultivar 'Tender and Soft as Water' were used in statistical analysis and verification of the accuracy of the regression models.

Received: 12 June 2020; Accepted: 18 January 2021

Published online: 03 February 2021

\section{References}

1. Irina, M., Martin, S. \& Joerg, F. Carbon transitions from either Calvin cycle or transitory starch to heteroglycans as revealed by 14C-labeling experiments using protoplasts from Arabidopsis. Physiol. Plant. 149, 25-44. https://doi.org/10.1111/ppl.12033 (2013).

2. Alison, M. S. \& Mark, S. Coordination of carbon supply and plant growth. Plant Cell Environ. 30, 1126-1149. https://doi. org/10.1111/j.1365-3040.01708.X (2007). 
3. Sala, A., Piper, F. \& Hoch, G. Physiological mechanisms of drought-induced tree mortality are far from being resolved. New Phytol. 186(2), 274-281. https://doi.org/10.1111/j.1469-8137.2009.03167.x (2010).

4. Chapin, F. S., Schulze, E. D. \& Mooney, H. A. The ecology and economics of storage in plants. Annu. Rev. Ecol. Syst. 21, 423-447. https://doi.org/10.1146/annurev.es.21.110190.002231 (1990).

5. Wang, S. G., Ding, Y. L., Lin, S. Y., Ji, X. \& Zhan, H. Seasonal changes of endogenous soluble sugar and starch in different developmental stages of Fargesia yunnanensis. J. Wood Sci. 62, 1-11. https://doi.org/10.1007/s10086-015-1521-8 (2016).

6. Wei, J., Wu, C. Y., Jiang, Y. \& Wang, H. L. Samplesethod. Food Sci. 35, 136-140. https://doi.org/10.7506/spkx1002-6630-201424026 (2014).

7. Zhang, C. et al. Estimation and visualization of soluble sugar content in oilseed rape leaves using hyperspectral imaging. Trans. ASABE 59, 1499-1505. https://doi.org/10.13031/trans.59.10485 (2016).

8. Li, Y., Mehta, R. \& Messing, J. A new high-throughput assay for determining soluble sugar in sorghum internode-extracted juice. Planta 248, 785-793. https://doi.org/10.1007/s00425-018-2932-8 (2018).

9. Webster, J. G. Electrical Impedance Tomography (Taylor and Francis Group, Oxford, 1990).

10. Wi, H., Sohal, H., McEwan, A. L., Woo, E. J. \& Oh, T. I. Multi-frequency electrical impedance tomography system with automatic self-calibration for long-term monitoring. IEEE Trans. Biomed. Circuits Syst. 8(1), 119-128. https://doi.org/10.1109/TBCAS .2013.2256785 (2014).

11. Bera, T, K., \& Nagaraju, J. A labVIEW based data acquisition system for electrical impedance tomography (EIT). In: Proceedings of the 3rd international conference on soft computing for problem solving. Springer India, (a): 377-389. https://doi.org/10.1007/97881-322-1768-8_34 (2014).

12. Bera, T. K., Nagaraju, J. \& Lubineau, G. Electrical impedance spectroscopy (EIS)-based evaluation of biological tissue phantoms to study multifrequency electrical impedance tomography (Mf-EIT) systems. J. Visual. 19(4), 691-713. https://doi.org/10.1007/ s12650-016-0351-0 (2016).

13. Ando, Y., Mizutani, K. \& Wakatsuki, N. Electrical impedance analysis of potato tissues during drying. J. Food Eng. 121(1), 24-31. https://doi.org/10.1016/j.jfoodeng.2013.08.008 (2014).

14. Kanti, B. T. Bioelectrical impedance methods for noninvasive health monitoring: a review. J. Med. Eng. https://doi. org/10.1155/2014/381251 (2014).

15. Chen, X. H. et al. Multi-channel electrical impedance tomography for regional tissue hydration monitoring. Physiol. Meas. 35(6), 1137-1147. https://doi.org/10.1088/0967-3334/35/6/1137 (2014).

16. Bayford, R. H. Bioimpedance tomography (electrical impedance tomography). Annu. Rev. Biomed. Eng. 8(1), 63-91. https://doi. org/10.1146/annurev.bioeng.8.061505.095716 (2006).

17. Bayford, R. \& Tizzard, A. Bioimpedance imaging: an overview of potential clinical applications. Analyst 137(20), 4635-4643. https ://doi.org/10.1039/C2AN35874C (2012).

18. Weigand, M. \& Kemna, A. Multi-frequency electrical impedance tomography as a non-invasive tool to characterize and monitor crop root systems. Biogeosciences 14, 921-939. https://doi.org/10.5194/bg-14-921-2017 (2017).

19. Mary, B. et al. Improvement of coarse root detection using time and frequency induced polarization: from laboratory to field experiments. Plant Soil. 417, 243-259. https://doi.org/10.1007/s11104-017-3255-4 (2017).

20. Martin, T. \& Günther, T. Complex resistivity tomography (CRT) for fungus detection on standing oak trees. Eur. J. For. Res. 132, 765-776. https://doi.org/10.1007/s10342-013-0711-4 (2013).

21. Jiao, M. L. et al. Frost hardiness assessment of floribunda roses by means of electrical impedance tomography. Scientia Agricultura Sinica 050(007), 1302-1316 (2017) (in Chinese).

22. Guyot, A. et al. Using electrical resistivity tomography to differentiate sapwood from heartwood: application to conifers. Tree Physiol. 33(2), 187-194. https://doi.org/10.1093/treephys/tps128 (2013).

23. Cole, K. S. \& Guttman, R. M. Electric impedance of frog egg. J. Gen. Physiol. 25(5), 765-775. https://doi.org/10.1085/jgp.25.5.765 (1942).

24. Yun, S. K. et al. Sugar, starch, and proline in peach trees exposed to freezing temperatures during dehardening. Agric. Sci. 05(10), 913-921 (2014).

25. Li, M. Q., Li, J. Y., Mao, H. P. \& Wu, Y. Y. Diagnosis and detection of phosphorus nutrition level for Solanum lycopersicum based on electrical impedance spectroscopy. Biosyst. Eng. 143, 108-118. https://doi.org/10.1016/j.biosystemseng.2016.01.005 (2016).

26. Liu, H., Fu, Y. M., Hu, D. W., Yu, J. \& Liu, H. Effect of green, yellow and purple radiation on biomass, photosynthesis, morphology and soluble sugar content of leafy lettuce via spectral wavebands "knock out". Sci. Hortic. 236, 10-17. https://doi.org/10.1016/j. scienta.2018.03.02 (2018).

27. Wei, K. L. et al. Relationship between optical properties and soluble sugar contents of apple flesh during storage. Postharvest Biol. Technol. 159, 111021. https://doi.org/10.1016/j.postharvbio.2019.111021 (2020).

28. Wang, J. W. et al. Influence of long-term cold storage on phenylpropanoid and soluble sugarmetabolisms accompanied with peel browning of 'Nanguo' pears duringsubsequent shelf life. Sci. Hortic. 260, 108888. https://doi.org/10.1016/j.scienta.2019.108888 (2020).

29. Keys, A. J. \& Leegood, R. C. Photosynthetic Nitrogen Assimilation and Associated Carbon and Respiratory Metabolism (Springer, Berlin, 2002). https://doi.org/10.1007/0-306-48138-3

30. Avi, G. et al. Influence of diurnal photosynthetic activity on the morphology, structure, and thermal properties of normal and waxy barley starch. Int. J. Biol. Macromol. 98, 188-200. https://doi.org/10.1016/j.ijbiomac.2017.01.118 (2017).

31. Run, T. H., Fu, G. R. \& Fang, W. H. The permeability biology of plant cell membrane was studied by electrical impedance spectroscopy. Guangdong Agric. Sci. 5, 142-145. https://doi.org/10.16768/j.issn.1004-874x.2013.05.006 (2013).

32. Repo, T. et al. Effects of freeze-thaw injury on parameters of distributed electrical circuits of stems and needles of Scots pine seedlings at different stages of acclimation. J. Exp. Bot. 45(6), 823-833. https://doi.org/10.1093/jxb/45.6.823 (1994).

33. Zhang, G. et al. Effects of soil temperature on bud break, shoot and needle growth, and frost hardiness in Pinus sylvestris var. mongolica saplings during dehardening. Acta Physiol Plant. 39, 169. https://doi.org/10.1007/s11738-017-2470-1 (2017).

34. Zhang, Y. \& Gao, L. Sensor-networked underwater target tracking based on grubbs criterion and improved particle filter algorithm. IEEE Access PP(99), 1 (2019).

\section{Acknowledgements}

We wish to thank Dr Feng Fu and his research team members for their excellent technical assistance. This research was supported by grants from the National Natural Science Foundation of China (31272190 awarded to G.Z.), The Financial Aid Project for the Introduction of Overseas Students in Hebei Province (C201838), Hebei Province Higher Education Science and Technology Research Project (QN2019090) and China Agriculture Research System (CARS-27). 


\section{Author contributions}

In this paper, J.Q. and G.Z. planned the project and was responsible for part of the manuscript writing and editing. J.Z. and B.D. contributed to sample preparation and methodology, Y.L., J.Z. and X.Y. scontributed to data analysis and interpretation of the results. All authors reviewed the manuscript.

\section{Competing interests}

The authors declare no competing interests.

\section{Additional information}

Correspondence and requests for materials should be addressed to J.Q.

Reprints and permissions information is available at www.nature.com/reprints.

Publisher's note Springer Nature remains neutral with regard to jurisdictional claims in published maps and institutional affiliations.

(c) (i) Open Access This article is licensed under a Creative Commons Attribution 4.0 International License, which permits use, sharing, adaptation, distribution and reproduction in any medium or format, as long as you give appropriate credit to the original author(s) and the source, provide a link to the Creative Commons licence, and indicate if changes were made. The images or other third party material in this article are included in the article's Creative Commons licence, unless indicated otherwise in a credit line to the material. If material is not included in the article's Creative Commons licence and your intended use is not permitted by statutory regulation or exceeds the permitted use, you will need to obtain permission directly from the copyright holder. To view a copy of this licence, visit http://creativecommons.org/licenses/by/4.0/.

(C) The Author(s) 2021 